\title{
Iterated stretching and multiple beads-on-a-string phenomena in dilute solutions of highly extensible flexible polymers
}

Mónica S. Oliveira and Gareth H. McKinley

Citation: Phys. Fluids 17, 071704 (2005); doi: 10.1063/1.1949197

View online: http://dx.doi.org/10.1063/1.1949197

View Table of Contents: http://pof.aip.org/resource/1/PHFLE6/v17/i7

Published by the American Institute of Physics.

\section{Related Articles}

A new instrument for dynamic helical squeeze flow which superposes oscillatory shear and oscillatory squeeze flow

Rev. Sci. Instrum. 83, 085105 (2012)

The effects of hydrodynamic interaction and inertia in determining the high-frequency dynamic modulus of a viscoelastic fluid with two-point passive microrheology

Phys. Fluids 24, 073103 (2012)

MHD free convection flow of a visco-elastic (Kuvshiniski type) dusty gas through a semi infinite plate moving with velocity decreasing exponentially with time and radiative heat transfer

AIP Advances 1, 022132 (2011)

Transitional flow of a non-Newtonian fluid in a pipe: Experimental evidence of weak turbulence induced by shearthinning behavior

Phys. Fluids 22, 101701 (2010)

Effects of viscoelasticity on the probability density functions in turbulent channel flow

Phys. Fluids 21, 115106 (2009)

\section{Additional information on Phys. Fluids}

Journal Homepage: http://pof.aip.org/

Journal Information: http://pof.aip.org/about/about_the_journal

Top downloads: http://pof.aip.org/features/most_downloaded

Information for Authors: http://pof.aip.org/authors

\section{ADVERTISEMENT}

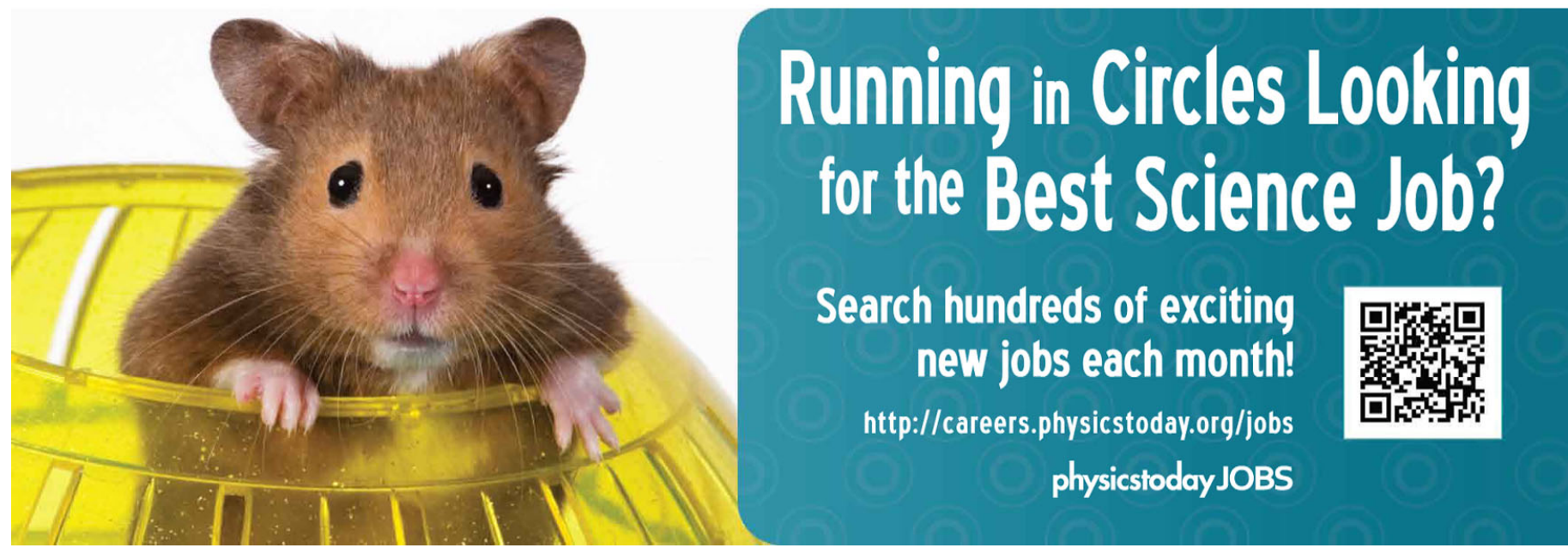




\title{
Iterated stretching and multiple beads-on-a-string phenomena in dilute solutions of highly extensible flexible polymers
}

\author{
Mónica S. N. Oliveira and Gareth H. McKinley \\ Hatsopoulos Microfluids Laboratory, Department of Mechanical Engineering, \\ Massachusetts Institute of Technology, Cambridge, Massachusetts 02139
}

(Received 2 March 2005; accepted 28 April 2005; published online 20 July 2005)

\begin{abstract}
The dynamics of elastocapillary thinning in high molecular weight polymer solutions are reexamined using high-speed digital video microscopy. At long times, the evolution of the viscoelastic thread deviates from self-similar exponential decay and the competition of elastic, capillary, and inertial forces leads to the formation of a periodic array of beads connected by axially uniform ligaments. This configuration is itself unstable and successive instabilities propagate from the necks connecting the beads and the ligaments. This iterated process results in the development of multiple generations of beads in agreement with the predictions of Chang, Demekin, and Kalaidin ["Iterated stretching of viscoelastic jets," Phys. Fluids 11, 1717 (1999)] although experiments yield a different recursion relation between successive generations. At long times, finite molecular extensibility truncates the iterated instability and axial translation of the bead arrays along the interconnecting threads leads to a progressive coalescence before the rupture of the filament.
\end{abstract}

(C) 2005 American Institute of Physics. [DOI: 10.1063/1.1949197]

It has been known for at least 40 years that the dynamics of capillary thinning and breakup of polymeric jets and threads are substantially different from the equivalent processes in Newtonian fluids. ${ }^{1,2}$ The capillary necking induced by surface tension results in a strong uniaxial stretching flow in the thread, which leads to a large molecular elongation and inhibits the finite time singularity associated with breakup in a Newtonian fluid jet. ${ }^{3,4}$ The large viscoelastic stresses ensuing from this stretching can also result in the formation of a characteristic morphology known as a beadson-a-string structure, in which spherical fluid droplets are interconnected by long thin fluid ligaments. Understanding the distribution of the droplets resulting from the dynamics of this process is important in numerous commercial applications including jet breakup, ${ }^{5}$ electrospinning, ${ }^{6}$ and inkjet printing (for further details see the monograph by Yarin ${ }^{7}$ ). Similar beads-on-a-string structures have also recently been documented in wormlike micellar solutions. ${ }^{8}$

The formation of a beads-on-a-string morphology is inherently a nonlinear dynamical process. Classical linear stability analysis shows that a viscoelastic fluid thread is in fact more unstable than a Newtonian fluid; however, uniaxial extensional flow in the filament results of exponential growth of the polymeric stresses and simulations show the formation of an axially uniform thread connecting two droplets. ${ }^{4,9}$ In the spherical beads, the molecules are relaxed and surface tension dominates; whereas in the thin ligament, the molecules are highly stretched and viscoelastic stresses dominate. Simple "zero-dimensional" analyses of this necking (in which axial variations of the thinning thread are neglected entirely) show that for an Oldroyd-B model the exponential growth of the elastic stresses is accompanied by an exponential decay in the filament radius: ${ }^{10}$

$$
R(t) / R_{P}=\left(\eta_{p} R_{P} / \lambda \sigma\right)^{1 / 3} \exp (-t / 3 \lambda)
$$

where $R_{P}$ is the plate radius, $\eta_{P}$ is the polymer viscosity, $\lambda$ is the relaxation time, and $\sigma$ is the surface tension. Direct measurement of this rate of decay thus enables quantitative determination of the fluid relaxation time..$^{10-13}$

Recent theoretical analyses and numerical simulations of one-dimensional slender filament models have shown that the profile $R(z, t)$ evolves in a self-similar manner; however, the precise dynamics depend on the relative magnitudes of the inertial, viscous, elastic, and capillary terms in the governing equations. ${ }^{14,15}$ Ultimately, finite extensibility of the molecules truncates the exponential stress growth, and the thread is then expected to thin linearly in time towards a breakup event with a general form $R(t) \sim\left(\sigma / \eta_{E}\right)\left(t_{c}-t\right)$. The magnitude of the extensional viscosity $\eta_{E}$ depends on the molecular weight of the polymer and the specific form of the nonlinear constitutive model. ${ }^{10,16,17}$ It is very hard to quantitatively evaluate this property for dilute polymer solutions using other techniques; ${ }^{18}$ however, monitoring the slow capillary drainage and ultimate rupture of a necking fluid thread can provide a suitable way of measuring this elusive material function. ${ }^{3,12,19}$ Recent numerical simulations have also demonstrated the evolution and the interaction of multiple beads on a viscoelastic thread. The slow drainage of fluid into the beads results in small, but nonzero, axial variations in the radii of the slender filaments between the beads. Imbalances in the magnitudes of the tensile stresses in the threads connected to the two poles of a bead lead to axial drainage and coalescence of beads along a thinning jet. ${ }^{20}$

Chang et $_{\text {al. }}{ }^{21}$ studied the dynamics of the slender filament equations for a finitely extensible nonlinear elastic (FENE) dumbbell model and predicted that at long times an additional phenomenon, termed "iterated stretching," should develop. In this regime, the neck connecting the cylindrical 
thread to the spherical bead was shown to be unstable to perturbations, which triggered a new instability and an "elastic recoil" close to the neck. This recoil leads to the formation of a new smaller "secondary" spherical drop connected to the primary drop by a thinner cylindrical thread. This new thread thins under the action of capillarity and the necks connecting the thread to the primary drop and to the new secondary drop may once again become unstable. This hierarchical process can repeat indefinitely, providing the molecules have not reached full extension, leading to multiple generations of beads on the strings. Iterated instabilities have been predicted numerically and observed experimentally in viscous fluid threads, ${ }^{22}$ but they do not lead to the formation of periodic structures such as beads-on-a-string. The initial elastic recoil and the formation of a secondary drop during pinchoff of a polymeric droplet have been documented by Cooper-White et al. ${ }^{23}$ but gravitational acceleration prohibits development of an iterated instability. In the present Letter, we demonstrate experimentally the phenomenon of iterated instability for the first time in a viscoelastic fluid thread undergoing capillary thinning.

The thinning of a polymer solution described by a FENE constitutive model is controlled by multiple physical parameters that can be combined to give four dimensionless parameters; a Deborah number defined as a ratio of the polymer relaxation time to the Rayleigh time scale for inertiocapillary breakup of a thread of radius $r_{0}, \mathrm{De}=\lambda / \sqrt{\rho r_{0}^{3} / \sigma}$; an Ohnesorge number characterizing the importance of viscous effects in the thread, $\mathrm{Oh}=\eta_{0} / \sqrt{\rho \sigma r_{0}}$; a solvent viscosity ratio $S=\eta_{s} /\left(\eta_{s}+\eta_{p}\right)=\eta_{s} / \eta_{0}$; and a finite extensibility parameter $L^{2}$ which characterizes the ratio of the maximum length to equilibrium length of the polymer molecules and scales with molecular weight of the solute, $L^{2} \sim M_{w}$. Here, $\rho$ is the density of the fluid, $\eta_{s}$ is the solvent viscosity, and $\eta_{0}$ is the total zero-shear-rate viscosity. All previous numerical predictions and experimental studies of capillary thinning and viscoelastic thread breakup can be represented in different regimes of this four-dimensional parameter space. In particular, Chang et al $^{21}$ demonstrated that for iterated stretching to be observed, one requires high Deborah numbers De $\gg 1$, intermediate viscosity ratios $(S \neq 0,1)$, finite fluid inertia $\mathrm{Oh} \sim O(1)$ - so that inertial effects lead to a rapid growth of the capillary instability and recoil—plus very high finite extensibilities $L^{2} \gg 1$, so that the iterated nature of the instability and elastic recoil process is not truncated prematurely by the maximum length of the molecules.

To obtain such values experimentally, we use a watersoluble flexible polymer, poly(ethylene oxide) (PEO), commonly used in viscoelastic jet breakup studies. ${ }^{5}$ The specific grade of polymer used (WSR-301) is commercially available and polydisperse, with a molecular weight $\overline{M_{w}} \approx 3.8$ $\times 10^{6} \mathrm{~g} / \mathrm{mol}$ and intrinsic viscosity $[\eta]_{0} \approx 1.42 \times 10^{3} \mathrm{~cm}^{3} / \mathrm{g}$. The polymer is dissolved $(C=2000 \mathrm{ppm})$ in a mixture of ethylene glycol and water to give a semidilute viscoelastic polymer solution with physical properties: $\eta_{s}=6.8 \mathrm{mPa} \mathrm{s}$, $\eta_{0}=47.1 \mathrm{mPa} \mathrm{s}, \sigma=0.0623 \mathrm{~N} \mathrm{~m}^{-1}$, and $\rho=925 \mathrm{~kg} \mathrm{~m}^{-3}$. The high molecular weight and the flexibility of the polymer chain indicate an extensibility $L^{2} \approx 2.4 \times 10^{4}$ and our measurements of the fluid relaxation time (described below)

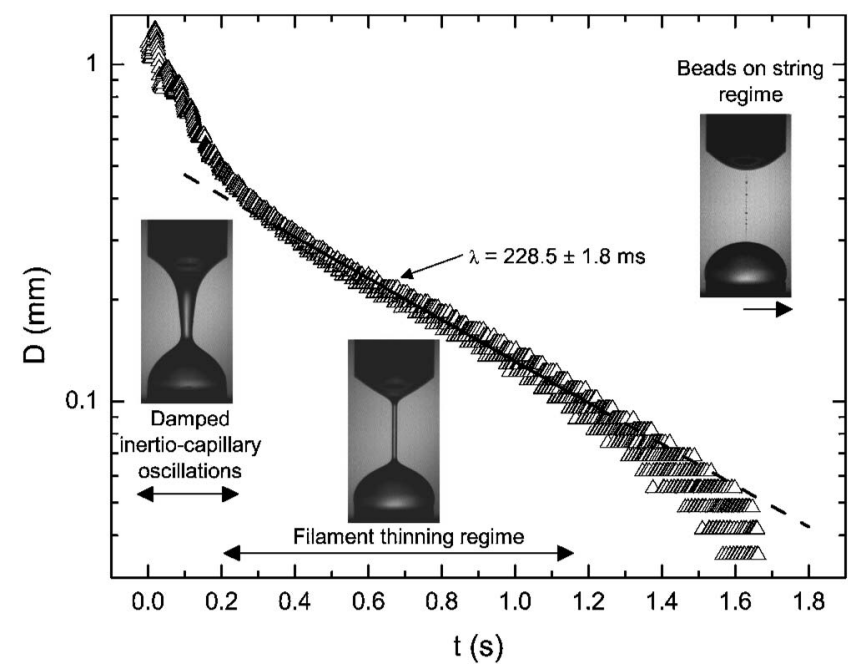

FIG. 1. Evolution of filament diameter undergoing CaBER. The symbols represent experimental data obtained using a laser micrometer; the solid line corresponds to the regression using Eq. (1).

show that the Deborah number in fluid threads of initial diameter $2 r_{0} \approx 1.2 \mathrm{~mm}$ is $\mathrm{De} \approx 127$. Eggers ${ }^{15}$ notes that inertial, viscous, and capillary effects will all become important in a necking fluid thread (such that $\mathrm{Oh} \sim 1$ ) on length scales $\ell \sim \eta_{0}^{2} /(\rho \sigma)$. For the present PEO solution, this corresponds to $\ell \approx 39 \mu \mathrm{m}$. We use a high-speed digital CMOS video camera (Phantom 5) operating at a frame rate of 1600-1800 fps in conjunction with a high-resolution video microscope lens system (Infinity K2) to resolve the late stage dynamics.

In Fig. 1, we show measurements of the global thinning dynamics in a capillary breakup extensional rheometer (CaBER-1, Cambridge Polymer Group). Initially, the $6 \mathrm{~mm}$ diameter plates are separated by a gap $h_{\mathrm{i}}=3 \mathrm{~mm}$. The liquid bridge confined between the plates is stretched as the top plate moves exponentially $(-50 \mathrm{~ms} \leqslant t \leqslant 0)$ to a specified distance $h_{0}=9.7 \mathrm{~mm}$. A laser micrometer (Omron ZL4-A) measures the evolution of the midpoint filament diameter $D(t)$ as the thread thins under the action of viscoelasticity and capillarity. A number of different regimes can be discerned in Fig. 1. Shortly after the top plate comes to a halt, inertiocapillary oscillations of the hemispherical fluid droplets attached to the end plates occur $(0 \leqslant t \leqslant 0.3 \mathrm{~s})$. These oscillations (with period $T \sim t_{\text {Rayleigh }}$ ) are damped by fluid viscosity and forthwith these regions act as quasistatic fluid reservoirs into which fluid from the necking thread can drain. The oscillations are followed by the rapid development of a central axially uniform filament of initial diameter $2 r_{0} \approx 0.4 \mathrm{~mm}$, which drains very slowly. On intermediate time and length scales, inertial, viscous, and gravitational effects can be neglected and a balance between surface tension and elasticity governs the filament drainage. ${ }^{10}$ In this regime, the local extensional rate in the filament is constant and the diameter decays exponentially with time according to Eq. (1) yielding $\lambda=229 \mathrm{~ms}$. The initial diameter of the elastic thread is also in approximate agreement with the theoretical expectation $^{21}\left(2 r_{0} / 2 R_{P}\right)_{\text {theory }}=\left(\eta_{p} R_{P} / \lambda \sigma\right)^{1 / 2}$, which 


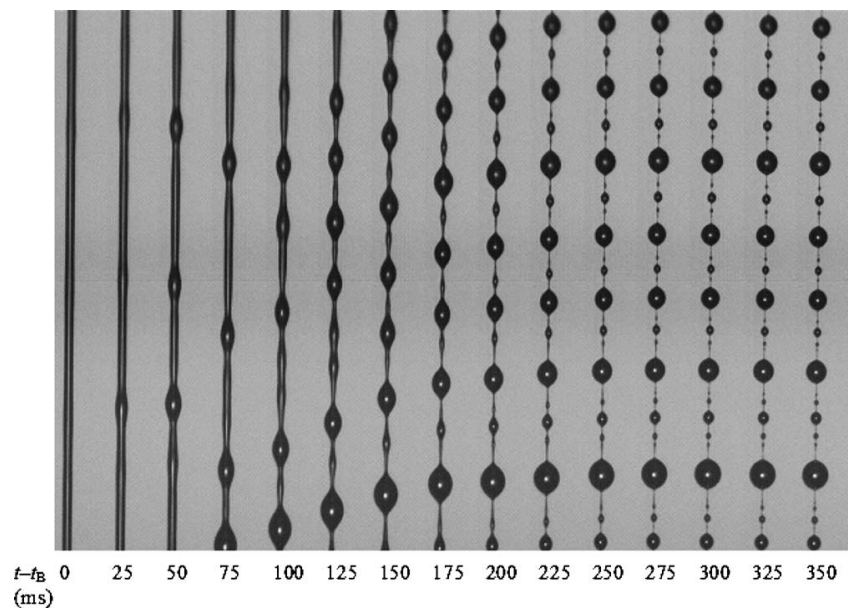

FIG. 2. Sequence of digital video images $\left(226 \times 2317 \mu \mathrm{m}^{2}\right)$ of the evolution of beads-on-a-string.

yields $\left(2 r_{0}\right)_{\text {theory }} \approx 0.56 \mathrm{~mm}$ for an initial plate radius of $R_{P}$ $=3 \mathrm{~mm}$.

At long times $t \geqslant t_{B} \approx 1.7 \mathrm{~s}(t / \lambda \geqslant 7.5)$, the necking thread approaches the characteristic length scale $\ell=39 \mu \mathrm{m}$ at which inertial, capillary, and viscous effects are all important. The inset video image in Fig. 1 shows that a series of regularly-spaced beads form on the viscoelastic fluid. The laser micrometer has a calibrated resolution of $20 \mu \mathrm{m}$ and the signal/noise ratio becomes increasingly poor at these scales. We, therefore, utilize the high-resolution digital video images for further analysis. A sequence of images showing the formation of the beads near the center of the filament is presented in Fig. 2. The iterated nature of the bead formation process is clear. Following the initial instability and the formation of a primary generation of beads, the new interconnecting fluid threads become unstable and form a second and third generation of beads. Detailed analysis of digitized profiles shows that as the primary bead forms, pinching occurs in the necks on each side of the bead. At this point, the filament at the neck is thinner than in the main thread away from the beads. The fluid gradually recoils and feeds a newly developing bead on each side of the primary drop. Meanwhile, the main filament connecting the beads grows thinner and the main bead collapses into an almost spherical shape. The process of pinching and recoiling can also be seen for subsequent generations. Using image analysis software, we are able to measure the diameters of the connecting filament at the onset of each bead formation event (denoted henceforth $D_{N}$, for $N=1,2, \ldots$ ), as well as the bead diameters for each successive generation. The fourth generation is just discernable but hard to quantify as the beads approach the minimum spatial resolution of the image $(1 \mathrm{pixel} \approx 2.3 \mu \mathrm{m})$. The data obtained are superimposed onto the laser micrometer measurements in Fig. 3. The formation and the growth of each new generation of beads is accompanied by a rapid thinning of the interconnecting filaments. These characteristics of the iterated stretching sequence are consistent with theoretical predictions. ${ }^{21}$ This analysis also predicted a recursive relationship between the filament diameters for successive generations. This is shown in the inset of Fig. 3 for the

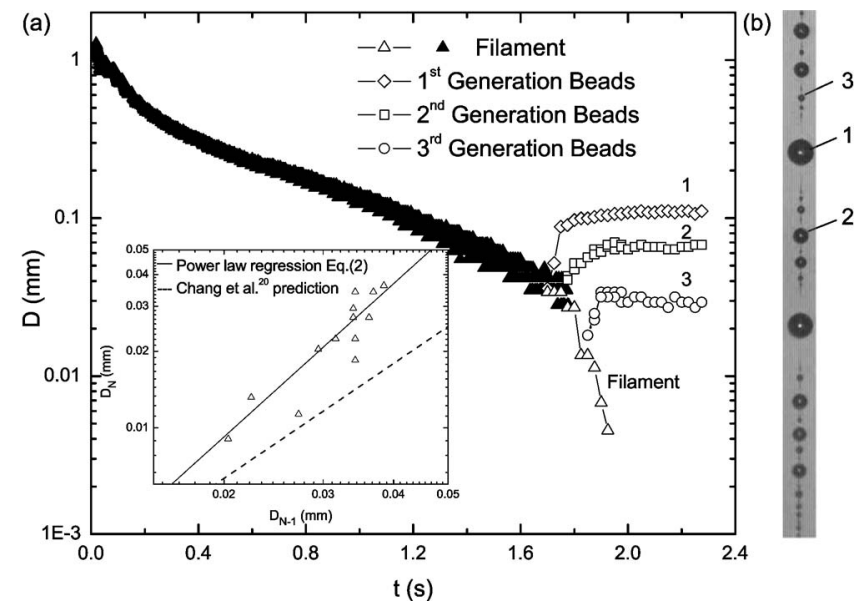

FIG. 3. (a) Evolution of the connecting filament ( $\mathbf{A})$ and beads (hollow symbols). The inset shows the recursive relationship between filament diameters of successive generations; dashed line corresponds to prediction of Chang et al. (Ref. 21); solid line is the best power law [Eq. (2)]. (b) Typical image $\left(138 \times 2317 \mu \mathrm{m}^{2}\right)$ at $t-t_{B}=500 \mathrm{~ms}$ used for image analysis.

first four generations formed in six different experimental realizations. The data appear to be fitted to a good approximation by a power law of form

$$
\left(D_{N} / D^{*}\right)=\left(D_{N-1} / D^{*}\right)^{m} \text {. }
$$

A least-squares fit of Eq. (2) to the experimental data (solid line) yields $m=2$ and a characteristic length scale $D^{*}$ $\approx 44 \mu \mathrm{m}$, which is very close to the Eggers length scale $\ell$ $\approx 39 \mu \mathrm{m}$, computed a priori. The measured data do not seem to follow the relationship $\left(D_{N} / D_{0}\right)=\sqrt{2}\left(D_{N-1} / D_{0}\right)^{3 / 2}$ proposed by Chang et al. ${ }^{21}$ for generations $N \geqslant 2$. The differences are most likely the result of finite extensibility effects as we discuss below.

In order to represent the complete spatial and temporal dynamics, from the first stages of droplet formation through coalescence until filament breakage, we process the stream of digital images to construct a "space-time" diagram ${ }^{24}$ (Fig. $4)$. For each frame $i(=1,2, \ldots, 2547)$ and each axial position

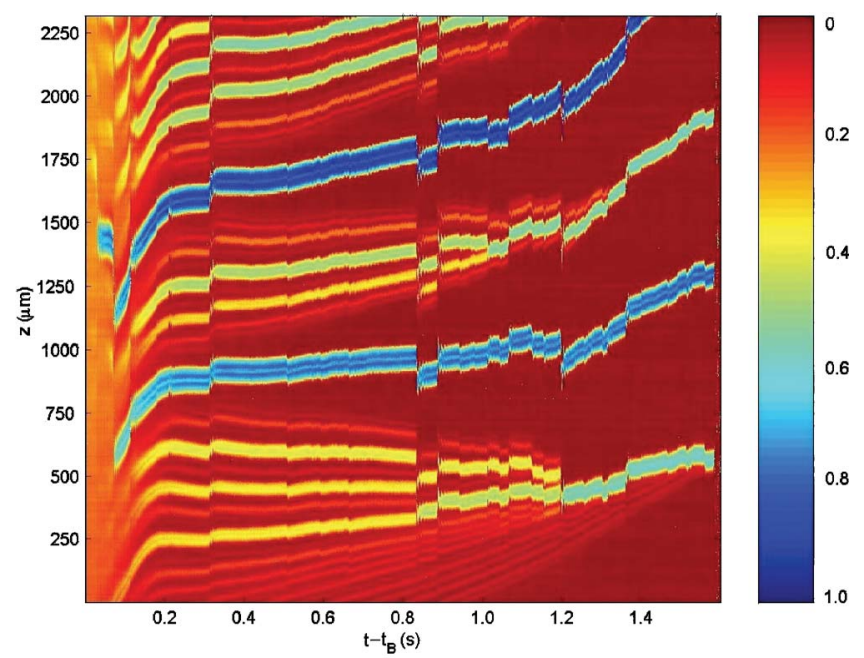

FIG. 4. (Color). Space-time diagram of the filament evolution. For each axial position $z$ and time $t-t_{B}$, the colors indicate relative thicknes ranging from zero filament thickness (dark red) to the thickest bead (dark blue). 
$z_{j}(1 \leqslant j \leqslant 1024)$, the average gray-scale intensity along the $x$ axis was calculated after background subtraction. Higher intensities correspond to thicker regions of fluid (e.g., beads), while low intensities correspond to thin fluid elements. The average intensities $\boldsymbol{I}\left(t_{\mathrm{i}}, z_{\mathrm{j}}\right)$ are rescaled from 0 to 1 , and the resulting image array then captures the evolution of the beads-on-a-string morphology. Initially, the intensity is homogeneous in $z$, showing the existence of a uniform filament. As the first generation of beads forms, we see the appearance of bright bands. Higher generations of beads are smaller and hence appear as progressively lower intensity traces. After about $0.5 \mathrm{~s}(\approx 2 \lambda)$, the beads-on-a-string structure is fully established and there is no visible formation of new beads. However, in contrast to the expected rupture event, ${ }^{2}$ we observe a new regime in which the fully formed beads migrate axially along the filament. As a result, coalescence between beads of different generations occurs and leads to a coarsening of the pattern. The creation of progressively larger beads is in accord with recent numerical descriptions of the draining and merging of beads. ${ }^{20}$ The large relief in elastic tension of the fluid thread following each coalescence event is evidenced by the very rapid axial displacements observed (e.g., at $t-t_{B}=0.82 \mathrm{~s}$ and $1.20 \mathrm{~s}$ ). Eventually, the structure has coarsened to a few large beads, the extensibility limit of the polymer is reached and the filament breaks.

Finally, we return to the use of elastocapillary thinning and breakup as an extensional rheometer. The balance of elastic and capillary forces in the axially uniform thread undergoing necking leads to an apparent extensional viscosity that is related to the first derivative of the filament diameter: $\eta_{\text {app }}=-\sigma /(d D / d t) .^{12}$ The resulting dimensionless Trouton ratio, $\operatorname{Tr}=\eta_{\text {app }} / \eta_{0}$, can then be evaluated as a function of the total Hencky strain, $\varepsilon_{H}=-2 \ln \left[D(t) / D_{P}\right]$. Space limitations preclude us from showing the data here; however, we find that the Trouton ratio initially grows exponentially and then approaches a steady state value at large Hencky strains $\left(\varepsilon_{H}\right.$ $\geq 8$ ). The asymptotic limit observed experimentally results in a very large ratio $\operatorname{Tr}_{\infty} \approx 1.3 \times 10^{4}$, which is in good agreement with the theoretical expectation for a dilute solution of FENE dumbbells, ${ }^{18,20} \operatorname{Tr}_{\infty} \rightarrow 2(1-S) L^{2} \approx 4.1 \times 10^{4}$.

Elastocapillary thinning and breakup thus provides a means of probing the transient extensional response even for very low viscosity - but highly elastic and extensiblepolymer solutions (i.e., viscoelastic fluids with $\mathrm{Oh} \ll 1$ but $\mathrm{De} \geqslant 1$ and $L^{2} \gg 1$ ). At late stages of thinning, such fluids are prone to iterated instabilities that result in an array of beadson-a-string and a subsequent slow axial drainage and consolidation phase. Most of the basic features we observe have been described in isolation by existing analyses; ${ }^{17,20,21}$ however, the interconnected nature of the exponential thinning, iterated instability, and coalescence phases has not been analyzed to date. We observe a different recursion relationship (inset in Fig. 3) to that obtained from an asymptotic analysis of the Oldroyd-B equation in the limit of infinite De. This difference in the observed scaling appears to be the result of an additional drainage of the interconnecting elastic threads between successive instability events coupled with the finite extensibility of the PEO chains. This is supported by a more detailed examination of the data which shows that the Trou- ton ratio is no longer climbing exponentially when the higher order generations of beads develop and the structure coarsens. It is to be hoped that these final stages of the drainage and breakup of polymer threads will be described by future analytic and numerical studies.

The authors would like to thank Professor E. S. G. Shaqfeh's research group and Fundação para a Ciência e Tecnologia (Portugal). They also would like to thank the reviewer for insightful comments.

${ }^{1}$ S. Middleman, "Stability of a viscoelastic jet," Chem. Eng. Sci. 20, 1037 (1965).

${ }^{2}$ M. Goldin, H. Yerushalmi, R. Pfeffer, and R. Shinnar, "Breakup of a laminar capillary jet of a viscoelastic fluid," J. Fluid Mech. 38, 689 (1969).

${ }^{3}$ Y. Amarouchene, D. Bonn, J. Meunier and H. Kellay, "Inhibition of the finite time singularity during droplet fission of a polymeric fluid," Phys. Rev. Lett. 86, 3558 (2001).

${ }^{4}$ M. Renardy, "A numerical study of the asymptotic evolution and breakup of Newtonian and viscoelastic jets," J. Non-Newtonian Fluid Mech. 59, 267 (1995)

${ }^{5}$ Y. Christanti and L. Walker, "Surface tension driven jet break up of strainhardening polymer solutions," J. Non-Newtonian Fluid Mech. 100, 9 (2001).

${ }^{6} \mathrm{H}$. Fong, I. Chun and D. H. Reneker, "Beaded nanofibers formed during electrospinning," Polymer 40, 4585 (1999).

${ }^{7}$ A. L. Yarin, Free Liquid Jets and Films: Hydrodynamics \& Rheology (Longman, New York, 1993).

${ }^{8}$ M. C. Sostarcez and A. Belmonte, "Beads-on-string phenomena in wormlike micellar fluids," Phys. Fluids 16, L67 (2004).

${ }^{9}$ D. W. Bousfield, R. Keunings, G. Marrucci and M. M. Denn, "Nonlinear analysis of the surface-tension driven breakup of viscoelastic fluid filaments," J. Non-Newtonian Fluid Mech. 21, 79 (1986).

${ }^{10} \mathrm{~V}$. M. Entov and E. J. Hinch, "Effect of a spectrum of relaxation times on the capillary thinning of a filament of elastic liquid," J. Non-Newtonian Fluid Mech. 72, 31 (1997).

${ }^{11}$ M. Stelter, G. Brenn, A. L. Yarin, R. P. Singh, and F. Durst, "Validation and application of a novel elongational device for polymer solutions," $\mathrm{J}$. Rheol. 44, 595 (2000).

${ }^{12}$ S. L. Anna and G. H. McKinley, "Elasto-capillary thinning and breakup of model elastic liquids," J. Rheol. 45, 115 (2001).

${ }^{13}$ A. V. Bazilevsky, V. M. Entov and A. N. Rozhkov, in Third European Rheology Conference, edited by D. R. Oliver (Elsevier Applied Science, Amsterdam, 1990), p. 41.

${ }^{14} \mathrm{M}$. Renardy, in Rheology Reviews, edited by D. M. Binding and K. Walters (The British Society of Rheology, Aberystwyth, 2004), Vol. 2, p. 171.

${ }^{15}$ J. Eggers, "Nonlinear dynamics and breakup of free-surface flows," Rev. Mod. Phys. 69, 865 (1997).

${ }^{16}$ M. Renardy, "Similarity solutions for jet breakup for various models of viscoelastic fluids," J. Non-Newtonian Fluid Mech. 104, 65 (2002).

${ }^{17} \mathrm{M}$. A. Fontelos and J. Li, "On the evolution and rupture of filaments in Giesekus and FENE models," J. Non-Newtonian Fluid Mech. 118, 1 (2004).

${ }^{18}$ A. Lindner, J. Vermant and D. Bonn, "How to obtain the elongational viscosity of dilute polymer solutions," Physica A 319, 125 (2003).

${ }^{19}$ M. Stelter, G. Brenn, A. L. Yarin, R. P. Singh, and F. Durst, "Investigation of the elongational behavior of polymer solutions by means of an elongational rheometer," J. Rheol. 46, 507 (2002).

${ }^{20} \mathrm{~J}$. Li and M. A. Fontelos, "Drop dynamics on the beads-on-string structure for viscoelastic jets: A numerical study," Phys. Fluids 15, 922 (2003).

${ }^{21}$ H. C. Chang, E. A. Demekhin, and E. Kalaidin, "Iterated stretching of viscoelastic jets," Phys. Fluids 11, 1717 (1999).

${ }^{22}$ M. P. Brenner, X. D. Shi and S. R. Nagel, "Iterated instabilities during droplet fission," Phys. Rev. Lett. 73, 3391 (1994).

${ }^{23}$ J. J. Cooper-White, J. E. Fagan, V. Tirtaatmadja, D. R. Lester and D. V. Boger, "Drop formation dynamics of constant low-viscosity, elastic fluids," J. Non-Newtonian Fluid Mech. 106, 29 (2002).

${ }^{24}$ B. M. Baumert and S. J. Muller, "Flow regimes in model viscoelastic fluids in a circular Couette system with independently rotating cylinders," Phys. Fluids 9, 566 (1997). 